\title{
Evaluation of Combined Energy-Efficient and Stability Strategies Utilising Direct Yaw Moment Control
}

\author{
Peikun Sun ${ }^{1(\otimes)}$, Annika Stensson Trigell ${ }^{1,2}$ Lars Drugge $^{1,2}$, \\ and Jenny Jerrelind ${ }^{1,2}$ \\ ${ }^{1}$ KTH vehicle dynamics, KTH Royal Institute of Technology, \\ 10044 Stockholm, Sweden \\ peikun@kth.se \\ 2 Centre for ECO2 Vehicle Design, KTH Royal Institute of Technology, \\ 10044 Stockholm, Sweden \\ https://www.kth.se/profile/peikun
}

\begin{abstract}
For sustainability reasons it is important to reduce energy consumption during driving. One contributionto energysavings is by using proper wheel torque distributions during manoeuvring. An active energy-efficient direct yaw moment control (DYC) for electric vehicles has previously been proposed by the authors, taking the motor efficiency map into consideration. The results show a potential for reduced energy losses during driving, but it might result in stabilityproblems during safety-critical maneuvres. In this work, consequences on stability due to this proposed energy efficient DYC is explored. Also an approach combining DYC both energy-efficiency and stability is proposed. The simulation results show that for the studied case the combination of DYC for energy-efficiency and stability can have an potential to both keep the vehicle safe and save considerable percentage of energy during both non safety-critical and safety-critical driving manoeuvres.
\end{abstract}

Keywords: Energy-efficient · Direct yaw moment control $\cdot$ Stability

\section{Nomenclature}

$\begin{array}{llll}m & \text { Vehicle mass } & I_{z} & \text { Yaw moment of inertia } \\ \psi & \text { Yaw angle } & h & \text { Height of CoG } \\ \delta_{f} & \text { Front steering angle } & t_{w} & \text { Vehicle track width } \\ l_{f} & \text { Distance from COG to front axle } & F_{x i} & \text { Longitudinal force } \\ l_{r} \quad \text { Distance from COG to rear axle } & F_{y i} & \text { Lateral force } \\ F_{a r} \quad \text { Aerodynamic resistance } & v_{x} \quad \text { Longitudinal velocity } \\ v_{y} \quad \text { Lateral velocity } & T_{i} \quad \text { Driving torque } \\ f_{r r} \quad \text { Rolling resistance coefficient } & I_{w} \quad \text { Rotational inertia of the wheel } \\ \omega_{i} \quad \text { Wheel angular velocity } & R_{0} \quad \text { Effective radius of the tyre } \\ \kappa_{i} \quad \text { Longitudinal slipratio } & a_{y} \quad \text { Lateral acceleration } \\ \beta \quad \text { Body slipangle } & C_{a r} \text { Aerodynamic resistance coefficient } \\ C_{f} \quad \text { Front axle cornering stiffness } & C_{r} \quad \text { Rear axle cornering stiffness } \\ (\text { CoG: centre of gravity) } & & \\ (i=1: \text { front left, } i=2: \text { front right, } i=3: & \text { rear left, } i=4: \text { rear right.) }\end{array}$




\section{Introduction}

Direct yaw moment control (DYC) of in-wheel motor vehicles can reduce energy consumption by means of proper wheel torque distribution, however, it usually is a passive way to save energy [1-3]. Although stability control is prioritized, during non safety-critical driving manoeuvres, energy-efficient driving should play the dominant role. An active energy-efficient DYC method taking into account the motor efficiency map was proposed in previous work [4]. In this paper, stability judgements consisting of yaw rate and body slip angle are designed and the switching principles betweenenergy-efficient DYC andstability DYC are devised. When the yaw rate and body slip angle are out of the set boundaries, stability control takes over energy-efficient DYC control until the working area is within the boundaries.

\section{Methods}

\subsection{Vehicle Model}

A two trackvehicle model is used and front wheel steering is adopted. The longitudinal, lateral and yaw motions are shown in Eqs. (1)-(3)

$$
\begin{gathered}
m\left(\dot{v}_{x}-v_{y} \dot{\psi}\right)=F_{x 1} \cos \delta_{f}+F_{x 2} \cos \delta_{f}+F_{x 3}+F_{x 4}-F_{y 1} \sin \delta_{f}-F_{y 2} \sin \delta_{f}-F_{a r} \\
m\left(\dot{v}_{y}+v_{x} \dot{\psi}\right)=F_{x 1} \sin \delta_{f}+F_{x 2} \sin \delta_{f}+F_{y 1} \cos \delta_{f}+F_{y 2} \cos \delta_{f}+F_{y 3}+F_{y 4} \\
I_{z} \ddot{\psi}=\left(F_{x 1} \sin \delta_{f}+F_{x 2} \sin \delta_{f}+F_{y 1} \cos \delta_{f}+F_{y 2} \cos \delta_{f}\right) l_{f}-\left(F_{y 3}+F y 4\right) l_{r} \\
+\left(F_{x 2} \cos \delta_{f}-F_{y 2} \sin \delta_{f}-F_{x 1} \cos \delta_{f}+F_{y 1} \sin \delta_{f}+F_{x 4}-F_{x 3}\right) t_{w} / 2
\end{gathered}
$$

The equation for the forward speed of each wheel can be expressed as

$$
\begin{cases}v_{1}=v_{x}-\dot{\psi} t_{w} / 2 ; & v_{2}=v_{x}+\dot{\psi} t_{w} / 2 \\ v_{3}=v_{x}-\dot{\psi} t_{w} / 2 ; & v_{4}=v_{x}+\dot{\psi} t_{w} / 2\end{cases}
$$

The angular velocity of the $i^{\text {th }}$ can be expressed as

$$
\omega_{i}=\left(1+\kappa_{i}\right) v_{i} / R_{0}
$$

The equation of tyre motion in the longitudinal direction can be written as

$$
T_{i}-f_{r r} F_{z i} R_{0}-F_{x i} R_{0}=I_{w} \dot{\omega}_{i}
$$

The aerodynamic resistance $F_{a r}$ can be expressed as

$$
F_{a r}=0.5 C_{a r} \rho A v_{x}^{2}
$$


The vehicle is designed to be a four-in-wheel motor electric vehicle and the chosenvehicle parameters are shown in Table 1. In the present study, Magic formula tyre models [5] for the longitudinal force and lateral force are used. Basedonthe characteristics of in-wheel motor data inChen [ 1], a simplified in-wheel motor efficiency map, shown in Fig. 1, is designed where the efficiency is a function of the motor torque.

Table 1. Vehicle parameters

\begin{tabular}{l|l|l|l|l|l|l|l}
\hline Parameter & Value & Parameter & Value & Parameter & Value & Parameter & Value \\
\hline$m$ & $1500 \mathrm{~kg}$ & $l_{r}$ & $1.5 \mathrm{~m}$ & $l_{f}$ & $1.2 \mathrm{~m}$ & $I_{z}$ & $1700 \mathrm{kgm}^{2}$ \\
\hline$t_{w}$ & $1.65 \mathrm{~m}$ & $I_{w}$ & $1 \mathrm{kgm}^{2}$ & $h$ & $0.48 \mathrm{~m}$ & $R_{0}$ & $0.3 \mathrm{~m}$ \\
\hline$C_{a r}$ & 0.3 & $A$ & $2 \mathrm{~m}^{2}$ & $f_{r r}$ & 0.01 & $C_{f}$ & $-87 \mathrm{kN} / \mathrm{rad}$ \\
\hline$C_{r}$ & $-69 \mathrm{kN} / \mathrm{rad}$ & & & & & & \\
\hline
\end{tabular}

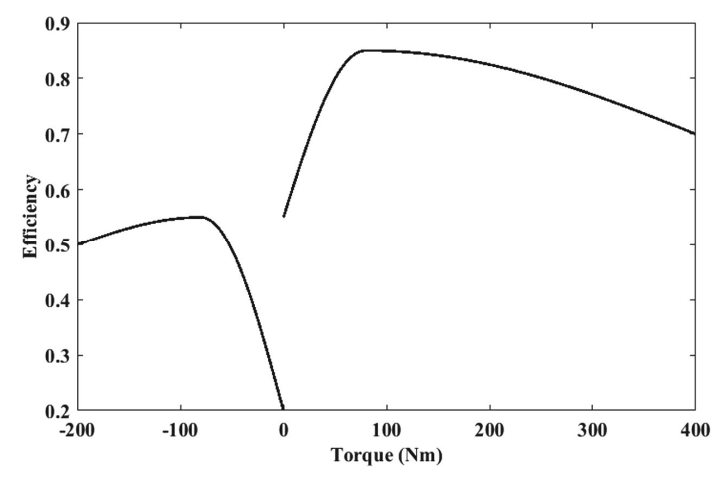

Fig. 1. The motor efficiency map

\subsection{Potential of DYC on Energy-SavingDuringSteady-State Cornering}

By analysing the vehicle characteristics in steady-state cornering, the fundamental vehicle motion characteristics can be understood [6]. The potential of DYC on energy-saving can be analysed through quasi-steady-state cornering when $\dot{v}_{y}=0, \ddot{\psi}=0$ and $\dot{\omega}_{i}=0$. During quasi-steady-state cornering, with a given $v_{x}, a_{y}$ and $M_{z}$, the total torque can be deduced. The offline optimisation can be developed where the procedure is shown in Fig. 2. Constant velocity cornering with $\dot{v}_{x}=0 \mathrm{~m} / \mathrm{s}^{2}$ and $v_{x}=80 \mathrm{~km} / \mathrm{h}$ is analysed. The range of $a_{y}$ is from $0 \mathrm{~m} / \mathrm{s}^{2}$ to $4 \mathrm{~m} / \mathrm{s}^{2}$ and for each $a_{y}, M_{z}$ is varied from $-1000 \mathrm{Nm}$ to $1000 \mathrm{Nm}$.

The optimisation results for $v_{x}=80 \mathrm{~km} / \mathrm{h}$ are shown in Figs. 3. In Fig. 3(a), two lateral accelerations results, $a_{y}=1 \mathrm{~m} / \mathrm{s}^{2}$ and $a_{y}=2 \mathrm{~m} / \mathrm{s}^{2}$, are presented. There are two local minimal power points for each $a_{y}$. In the $a_{y}$ range from $0 \mathrm{~m} / \mathrm{s}^{2}$ 


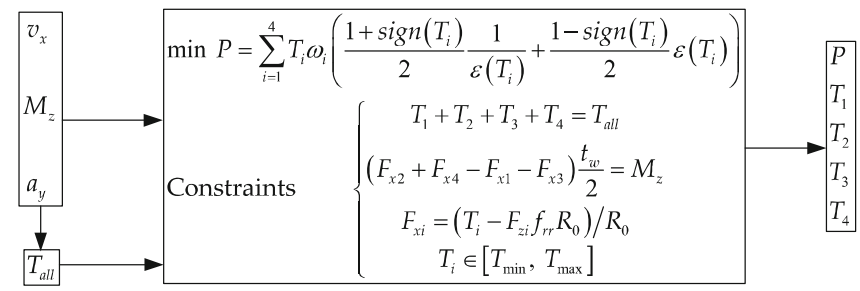

Fig. 2. The minimal power optimisation procedure under $M_{z}$

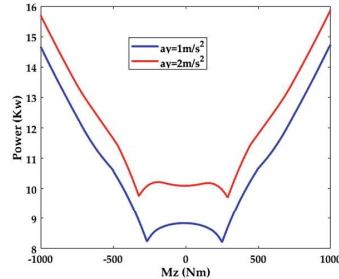

(a)

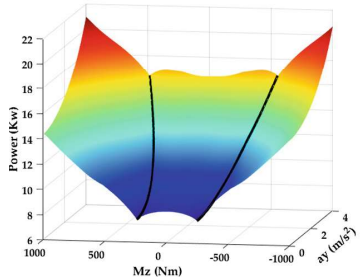

(d)

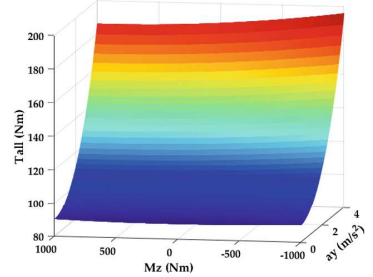

(b)

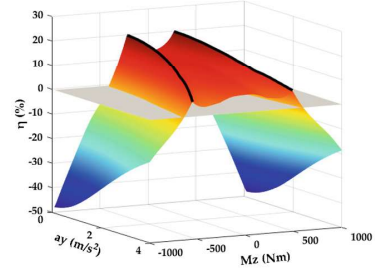

(e)

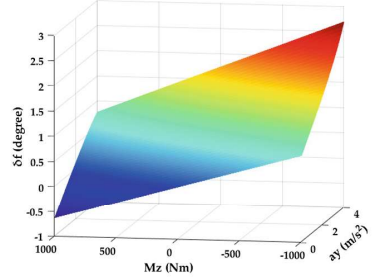

(c)

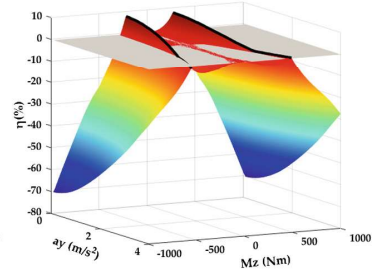

(f)

Fig. 3. Optimisation results for $v_{x}=80 \mathrm{~km} / \mathrm{h}$ : (a) Power versus $M_{z}$ for $a_{y}=1 \mathrm{~m} / \mathrm{s}^{2}$ and $a_{y}=2 \mathrm{~m} / \mathrm{s}^{2}$; (b) Total torque versus $a_{y}$ and $M_{z} ;$ (c) $\delta_{f}$ versus $a_{y}$ and $M_{z} ;$ (d) Power consumption versus $a_{y}$ and $M_{z}$; (e) Percentage of energy saving compared to four-wheel equal torque driving; (f) Percentage of energy saving compared to two-wheel equal torque driving.

to $4 \mathrm{~m} / \mathrm{s}^{2}$, the black lines in Fig. 3(d) represent local minimal power points. It can be seen in Fig. 3(e) and (f) that considerable percentage of energy saving can be achieved at local minimal power points compared to both two-wheel equal torque driving and four-wheel equal torque driving. The grey planes in Fig. 3(e) and (f) represent $0 \%$ energy saving. From Fig. 3(b), for each $a_{y}, M_{z}$ does not change $T_{\text {all }}$ so much. From Fig. 3 , it is shown that DYC can be used to reduce the power consumption.

\subsection{Energy-Efficient and StabilityDYC Design}

The idea of active energy-efficient DYC is to find the most energy-efficient yaw moment for a given reference lateral acceleration $a_{y r}$. The steering angle follows 


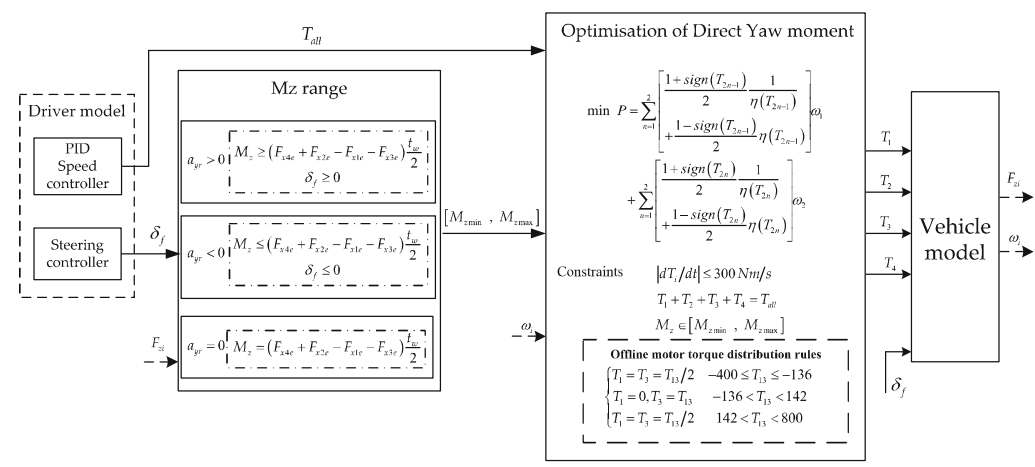

Fig. 4. The controller structure for energy-efficient DYC [4]

the same direction of $a_{y r}$ to avoid counter-steering. The offline torque distribution rule is optimised based on the motor efficiency map in Fig. 1. The structure of energy-efficient DYC is shown in Fig. 4.

The principle of stability DYCis that the desired yaw rate should be tracked. The reference yaw rate $\dot{\psi}_{r}[6]$ as a function $\delta_{f}$ can be expressed as Eq. 8 .

$$
\dot{\psi}_{r}=\frac{v_{x}}{L+\frac{m v_{x}^{2}}{L}\left(\frac{l_{f}}{C_{r}}-\frac{l_{r}}{C_{f}}\right)} \delta_{f}
$$

The stability yaw moment $M_{z s}$ is then

$$
M_{z s}=I_{z} \ddot{\psi}_{r}-\left(\left(F_{y 1}+F_{y 2}\right) l_{f}-\left(F_{y 3}+F_{y 4}\right) l_{r}\right)
$$

The vehicle stability working area can be a combination of the yaw rate $\dot{\psi}$ and the body slip angle $\beta$. In Rajamani [7], the simplified suggested ranges for yaw rate and body slip angle are expressed as

$$
\left\{\begin{array}{l}
|\dot{\psi}| \leq 0.85 \frac{\mu g}{v_{x}} \\
|\beta| \leq \operatorname{atan}(0.02 \mu g)
\end{array}\right.
$$

The $\dot{\psi}-\beta$ phase-plane method [8,9] and Lyapunov method [10] can also divide the vehicle working area into a stable and a non-stable region. The comparison of simplified method, phase-plane method and Lyapunov method are shown in Fig. 5 .

It can be seen that the simplified working area in Eq. (10) is within the phaseplane method and the Lyapunov method results in unreasonable slip angle and yawrate. Therefore, the simplified stability area described in Eq. (10) was chosen for the present study. 


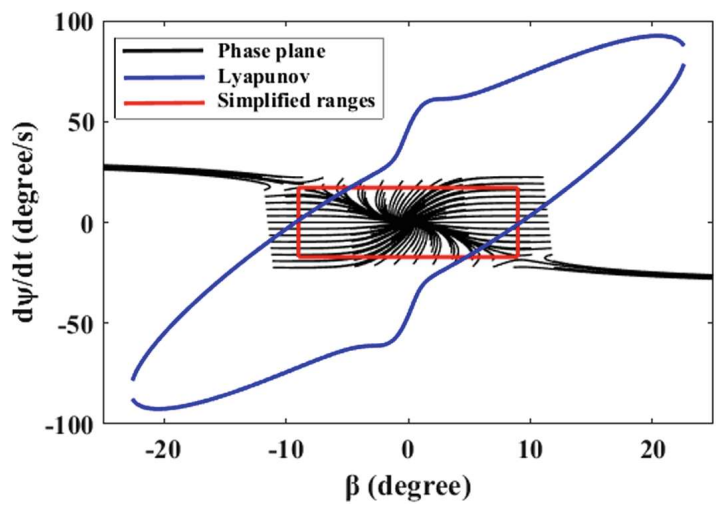

Fig. 5. Comparison of stability regions for $v_{x}=80 \mathrm{~km} / \mathrm{h}$ and $\mu=0.8$.

Thestrategy for both energy-efficient and stability DYC is that during nonsafety-cirtical manoeuvres, energy-efficient DYC plays the dominant role and during safety-critical manoeuvres, stability DYC control takes charge. The switching principle betweenenergy-efficient DYC andstability DYC is: when bothof $\dot{\psi}$ and $\beta$ are within0.6 times maximum values, energy-efficient DYC works; when any of $\dot{\psi}$ and $\beta$ is out of 0.6 times maximum values, stability DYC will be used until both of $\dot{\psi}$ and $\beta$ are within 0.58 times maximum values.

\section{Results andConclusions}

The ISO 3888-1 double lane change [11], shown in Fig. 6, is used to verify the effectiveness of the controller during transient cornering. For comparison, based on ISO 3888-1, an extended double lane change is designed. The path parameters are shown in Table 2.

Table 2. Path parameters

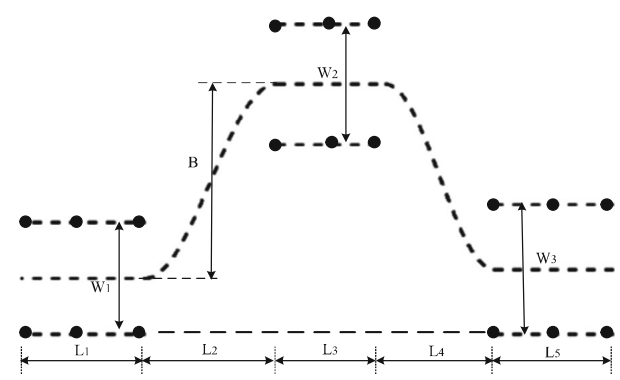

\begin{tabular}{l|l|l}
\hline Parameter & Extended & ISO3888-1 \\
\hline$L_{1}(\mathrm{~m})$ & 15 & 15 \\
\hline$L_{2}(\mathrm{~m})$ & 60 & 30 \\
\hline$L_{3}(\mathrm{~m})$ & 25 & 25 \\
\hline$L_{4}(\mathrm{~m})$ & 50 & 25 \\
\hline$L_{5}(\mathrm{~m})$ & 30 & 30 \\
\hline$W_{1}(\mathrm{~m})$ & 2.065 & 2.065 \\
\hline$W_{2}(\mathrm{~m})$ & 2.3125 & 2.3125 \\
\hline$W_{3}(\mathrm{~m})$ & 2.395 & 2.395 \\
\hline$B(\mathrm{~m})$ & 3.58 & 3.58 \\
\hline
\end{tabular}

Fig. 6. The double lane change path 
Table 3. Average power for all simulated manoeuvres

\begin{tabular}{l|c|l}
\hline Average power $(\mathrm{kW})$ & Extended & ISO3888-1 \\
\hline Four-wheel equal torque & 10.26 & 13.22 (unsafe) \\
\hline Two-wheel equal torque & 8.89 & 11.69 (unsafe) \\
\hline Energy-efficient DYC & 8.52 & 11.65 (unsafe) \\
\hline Stability DYC & 8.74 & 12.57 \\
\hline Combined DYC & 8.52 & 11.84 \\
\hline
\end{tabular}

Constant velocity $v_{x}=80 \mathrm{~km} / \mathrm{h}$ during these two paths are simulated and the results are shown in Figs. 7 and 8. The line colors are explained as follows: black: two-wheel equal torque driving; green: energy-efficient DYC; red: stability DYC; blue: combined DYC. The combined DYC is a combination of the proposed energy-efficient and stability controls. The average power consumptions of four-wheel equal torque driving, two-wheel equal torque driving, energy-efficient DYC, stability DYC and combined DYC are summarised in Table 3.

From Figs. 7(b), 8(b) and (e), when the lane change length reduces, the working area increases while using two-wheel equal torque driving and energy-efficient DYC. During the extendeddouble lane change inFig. 7, the working area is within the stability boundaries and the combined control is energy-efficient only. During ISO 3888-1 double lane change, only stability DYC and combined DYC can keep the working area within the stability boundaries. From Figs. 7(c), 8(c) and Table 3, it can be seen that the energy-efficient DYC during extended double lane change consumes the minimal power. Energy-efficient DYC can save 2.5\% energy compared to stability DYC, $4.1 \%$ compared to two-wheel equal torque driving and $16.9 \%$ compared to four-wheel equal torque driving. During the ISO 3888-1 double lane change, combined DYC can keep vehicle safe and can save $5.8 \%$ compared to stability DYC.

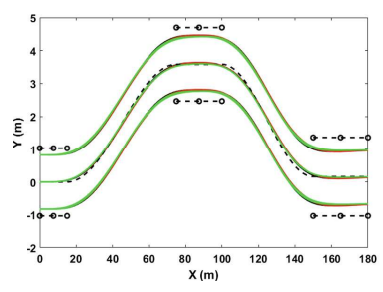

(a) Path

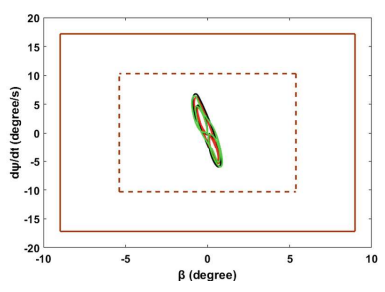

(b) Working area

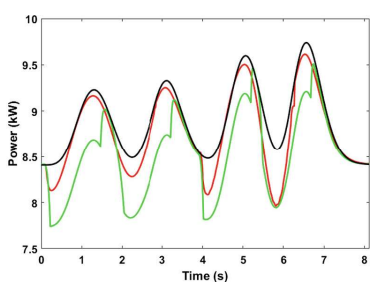

(c) Power

Fig. 7. Simulation results of extended double lane change 


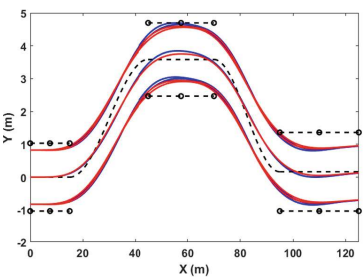

(a) Path

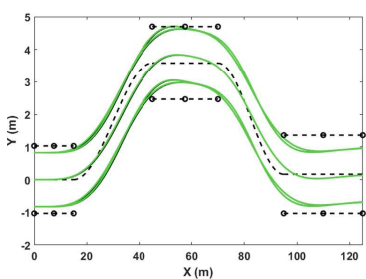

(d) Path

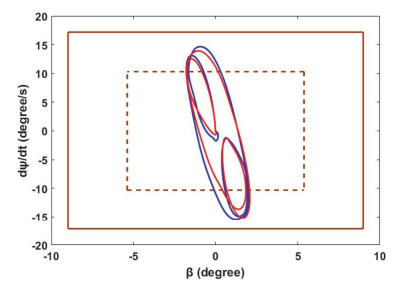

(b) Working area

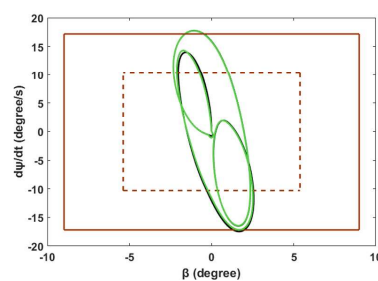

(e) Working area

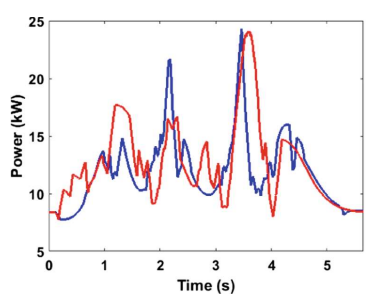

(c) Power

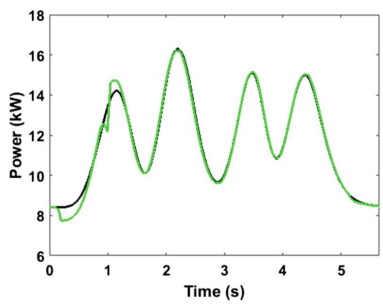

(f) Power

Fig. 8. Simulation results of ISO 3888-1 double lane change

Acknowledgment. The authors greatlyappreciate the support from China Scholarship Council (CSC), the Centre for ECO2 Vehicle Design at KTH funded by the Swedish Innovation AgencyVinnova (Grant Number 2016-05195) and the strategic research area TRENoP.

\section{References}

1. Chen, Y., Wang, J.M.: Adaptive energy-efficient control allocation for planar motion control of over-actuated electric ground vehicles. IEEE Trans. Control Syst. Technol. 22(4), 1362-1373 (2014). https://doi.org/10.1109/TCST.2013.2287560

2. Han, Z.L., Xu, N., Chen, H., Huang, Y.J., Zhao, B.: Energy-efficient control of electric vehicles based on linear quadratic regulator and phase plane analysis. Appl. Energy 213, 639-657 (2018). https://doi.org/10.1016/j.apenergy.2017.09.006

3. Zhai, L., Hou, R.F., Sun, T.M., Kavuma, S.: Continuous steering stability control based on an energy-saving torque distribution algorithm for a four in-wheel-motor independent-drive electric vehicle. Energies 11(2), 350 (2018). https://doi.org/10. 3390/en11020350

4. Sun, P., Stensson Trigell, A., Drugge, L., Jerrelind, J.: Energy-efficient direct yaw moment control for in-wheel motor electric vehicles utilising motor efficiency maps (2019). (Submitted for publishing)

5. Pacejka, H.: Tire and Vehicle Dynamics, 3rd edn. Elsevier, Amsterdam (2012)

6. Abe, M.: Vehicle Handling Dynamics: Theory and Application. Butterworth Heinemann, Amsterdam (2015)

7. Rajamani, R.: Vehicle Dynamics and Control. Springer, Boston (2012). https:// doi.org/10.1007/978-1-4614-1433-9

8. Bobier, C.G., Gerdes, J.C.: Staying within the nullcline boundary for vehicle envelope control using a sliding surface. Veh. Syst. Dyn. 51(2), 199-217 (2013) 
9. Ono, E., Hosoe, S., Tuan, H.D., Doi, S.: Bifurcation in vehicle dynamics and robust front wheel steering control. IEEE Trans. Control Syst. Technol. 6(3), 412-420 (1998)

10. Yin, G., Jin, X., Qing, Z., Bian, C.: Lateral stability region conservativeness estimation and torque distribution for FWIA electric vehicle steering. Sci. China Technol. Sci. 58(4), 669-676 (2014)

11. Passenger cars - Test track for a severelane-change manoeuvre - Part 1: Double lane-change, International Standards Organization (ISO) (2018). ISO: 888$1: 2018(\mathrm{E})$ 